\title{
One Avatar, Two Level of Detail, One Result? - Analyzing the Effect of Low and High Detailed Avatars on Fitting Simulations
}

\author{
Christian PIRCH, Oliver KAUSCH, Anke KLEPSER, Simone MORLOCK \\ Hohenstein Laboratories GmbH \& Co. KG, Bönnigheim, Germany
}

https://doi.org/10.15221/21.46

\begin{abstract}
3D garment simulation is in the market for many years. In the beginning, most users found it hard to transfer their traditional analogue processes into the digital world [1]. Yet, the technical enhancements of the simulation software fastened the readiness of the companies to implement systems in their product development cycle. Through the use of 3D garment simulation systems, resources can be saved and development times shortened [2-4]. In addition, garment simulation can already be used to check the fit in a digital environment. By being able to check design and fit early in the process, more time can be invested in their development. A complete simulation is important for a quality fit. In addition to the pattern, this also includes the material parameters and a digital fitting tape. For reliable fitting tests an avatar has to fulfil various requirements. This also includes the level of detail (LOD) of the avatar to perform valid quality assessments. It is assumed that the LOD has a major impact on fitting results [4] Yet, creating high level avatars require time and comprehensive knowledge of 3D workflows.
\end{abstract}

Keywords: 3D Scanning, 3D Fashion Simulation, 3D Avatar, Fitting, Garment

\section{Introduction}

Digital fitting models for virtual fit testing can, be generated in the garment simulation systems themselves via measurement controllers. The change of measurements often follows a standard measurement procedure, such as Size GERMANY [5] or the ASTM standard [6]. However, the body shape is not only influenced by changing the measurements; the actual shape and form of the various body regions can only be influenced in VStitcher [7] and there only to a limited extent. By creating avatars in the garment simulation systems, it is possible to work with fit dummies these correspond to the physical idealised fit busts and can also be used digitally $[8,9]$. Another possibility is to create avatars via editors without direct reference to the garment industry, such as Daz3D, Metahuman Creator or Make Human [10-12]. These tools offer a variety of possibilities to customise the avatar via textures and assets for the face. However, the avatar geometry is usually created using the body shape without dimensioning or specifying a measurement system. To create an avatar with the desired dimensions and body shape, another very precise way is to scan a test object. Depending on the precision and resolution, such a scan can consist of a networked point cloud of more than 1,000,000 points. The polycount is decisive for the performance of the garment simulation. The higher the polycount in the simulation, including clothing and avatar, the longer the calculation takes. Therefore, scan geometries are usually post-processed, within this process irregularities generated by the scanner are compensated and the point count is reduced by a retopology. There is no exact description of a workflow for creating an avatar geometry for a clothing simulation. However, there are some recommendations from VStitcher, such as the polycount, which should be between 20,000 and 100,000 [13].

In this research work the influence of the polycount on the clothing simulations in the systems Clo3D, VStitcher and Vidya will be investigated. For this purpose, the systems are considered individually without comparing the results. 


\section{Method}

\subsection{Avatar Creation and Investigation}

For the test series, a base geometry was created in the 3D software Blender on the basis of a scan in a relaxed posture of a female test person in size 50 according to SizeGERMANY. This base geometry corresponds to Level of Detail 0 and consists of a quadrilateral mesh that was projected onto the surface of the scan and aligned using anthropometric points. The base geometry was divided into several objects, on the one hand a coherent body geometry and on the other hand several objects that make up attributes in the face, such as eyes, eyelashes, eyebrows and teeth. The body geometry was further segmented into the groups head, body, arms, legs, hands and feet (see fig 1). The division of the basic geometry into further segments was necessary for the mesh analysis.

\section{Subdivison Modifier}

The further levels of detail were created with a subdivision modifier. But only for the body geometry, the geometries for the face details were not subdivided further. With this modifier the squares of the basic geometry were divided, through each level one square is divided into 4 more squares. Two methods can be chosen for this, the Simple Subdivision and the Catmull Clark method. In the first, the surfaces were only divided, and the corner points do not change their position. In the second method, the positions of the corner points are recalculated, resulting in smoother transitions between the resulting surfaces.

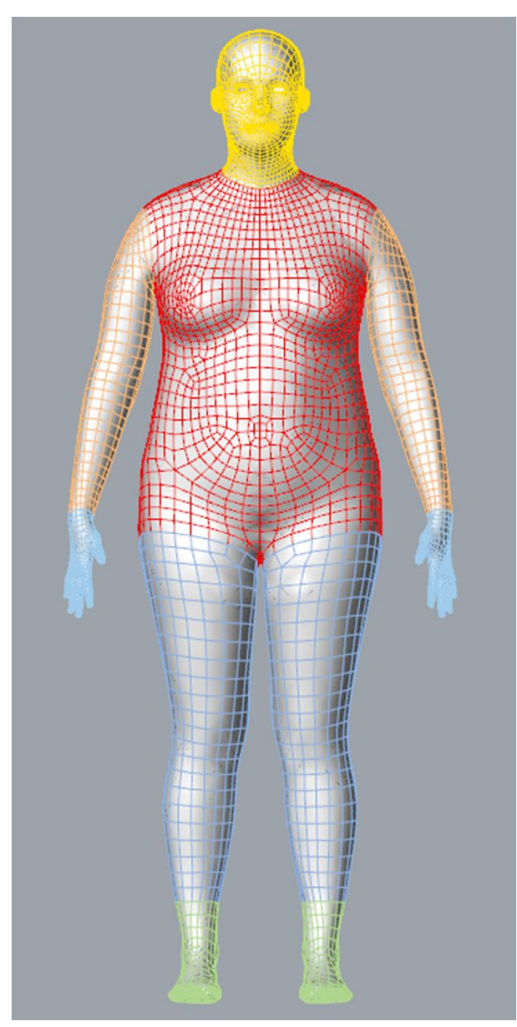

Fig. 1 Segmentation of the Base Geometry

In this test series, two further levels of detail were created in addition to Level of Detail 0 . In Level of Detail 1 the avatar was subdivided with a simple subdivision modifier and with the Catmull Clark procedure, in both the vertices were not realigned on the scan geometry. In LOD1.3, however, the base geometry on the scan geometry was then adjusted by reprojecting the vertices. In Level of Detail 2 , the base geometry was subdivided twice, in LOD2.1 using the Catmull Clark method and additionally reprojected in LOD2.2 (see table 1).

Table 1. Test Setup

$\begin{array}{llll}\text { Label } & \begin{array}{l}\text { Subdivision } \\ \text { Level }\end{array} & \text { Method } & \text { Reprojection } \\ \text { LOD0 } & 0 & - & - \\ \text { LOD1.1 } & 1 & \text { Simple Subdivision } & - \\ \text { LOD1.2 } & 1 & \text { Catmull Clark method } & - \\ \text { LOD1.3 } & 1 & \text { Simple Subdivision } & \text { Yes } \\ \text { LOD2.1 } & 2 & \text { Catmull Clark method } & - \\ \text { LOD2.2 } & 2 & \text { Simple Subdivision } & \text { Yes }\end{array}$

The six avatars created in the test series were investigated in a Four-step analysis. Firstly, the polycount of the created avatars was counted and compared. Then the main circumferences were measured and compared. The circumference measurements taken were bust circumference, waist circumference and hip circumference. In a further step, the topology of the geometries was examined more closely. This means the polycount and the structure of the surfaces in relation to each other were investigated. Lastly, the results of the clothing simulation were reviewed and compared to the topology of the avatar. 


\subsection{Garment Fit Inspection}

After this analysis, the geometries were then exported and imported into three garment simulation systems, Clo3D, Vidya and VStitcher systems and saved as avatars. A pair of trousers was then simulated on the different avatars. The standard materials pre-set in the systems were used for the simulation. The same positioning of the garments in the different simulations was given by lines on the texture of the avatar.
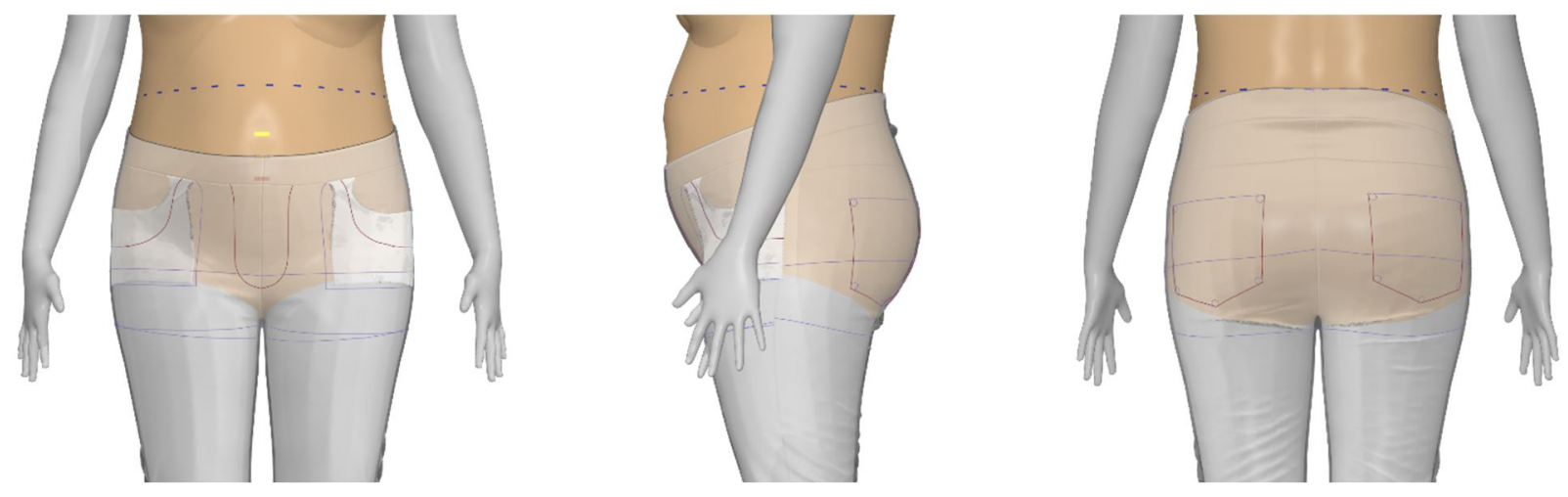

Fig. 2 Positioning of the pants

The trousers were positioned in the middle between the two colour-marked stripes at the front and on the dashed line at the back, no further action is taken to position the trousers beyond this (see fig 2).

Table 2 Fitting Simulation Settings

$\begin{array}{llll} & \text { Vidya } & \text { Clo3D } & \text { Vstitcher } \\ \text { Mesh density in } \mathbf{~ m m} & 7.36 & 5 & 2 \\ \text { Simulation algorithm } & \text { Precise collision detection } & \text { Fitting } & \text { Dress }\end{array}$

The smallest selectable mesh density in Vidya for the pant pattern pieces was 25, this corresponds to a mesh density of $7.36 \mathrm{~mm}$. After completing the simulation, the avatar was changed, the next avatar with a different Level of Detail was than imported. The garment is transferred to its pre-simulation positioning so that the position of the previous simulation does not influence it. Afterwards, the simulations on the three different avatars are compared in terms of their fit. The fit assessment is performed in two steps. First, the visual look of the garment was checked, and in the next step a structural fit analysis was carried out based on the analysis tools available from the programmes. The results of the fit assessment were then compared to those of the structural analysis of the geometries of the avatars.

\section{Results}

\subsection{Geometry}

Subdividing the avatar geometries resulted in the following polycounts for the new LODs (see Tab. 3). The polycount of more than 300.000 in LOD2 corresponds to an increase of more than tenfold from LOD0 and is thus four times higher than the recommendation of VStitcher Browzwear furthermore LOD1 with a polycount of more than 96.000 is about $11 \%$ higher than this recommendation. In addition, the results of the analysis show that the geometry of small body parts, e.g. hands and feed, are shaped with significant larger polycount than larger parts. Changing the LOD leads in all body areas to an increase of polygons, yet the relation remains stable.

\begin{tabular}{|c|c|c|c|c|c|c|c|c|c|}
\hline & $\begin{array}{l}\text { Polys } \\
\text { Body }\end{array}$ & $\begin{array}{l}\text { Polys } \\
\text { Head }\end{array}$ & $\begin{array}{l}\text { Polys } \\
\text { Body }\end{array}$ & $\begin{array}{l}\text { Polys } \\
\text { Arms }\end{array}$ & $\begin{array}{l}\text { Polys } \\
\text { Hands }\end{array}$ & $\begin{array}{l}\text { Polys } \\
\text { Legs }\end{array}$ & $\begin{array}{l}\text { Polys } \\
\text { Feet }\end{array}$ & $\begin{array}{l}\text { Polys } \\
\text { Face }\end{array}$ & $\begin{array}{l}\text { Polys } \\
\text { TOTAL }\end{array}$ \\
\hline LODO & 24.152 & 4.038 & 3.394 & 1.776 & 8.342 & 1.652 & 4.950 & 14.696 & 38.848 \\
\hline LOD1 & 96.968 & 16.512 & 13.576 & 7.104 & 33.368 & 6.608 & 19.800 & & 111.664 \\
\hline LOD2 & 386.436 & 64.608 & 54.304 & 28.416 & 133.476 & 26.432 & 79.200 & & 401.132 \\
\hline
\end{tabular}


The next step was to check how the circumferential dimensions of the geometries changed over the series of avatars.

Table 4. Circumference Measurements

\begin{tabular}{lllllll} 
& \multicolumn{2}{c}{ Bust Circumference } & \multicolumn{2}{c}{ Waist Circumference } & \multicolumn{2}{c}{ Hip Circumference } \\
Label & Measured & Difference & Measured & Difference & Measured & Difference \\
Scan & 117,73 & - & 103,04 & - & 119,30 & - \\
LOD0 & 117,36 & 0,37 & 102,94 & 0,10 & 119,09 & 0,21 \\
LOD1.1 & 117,37 & 0,36 & 102,94 & 0,10 & 119,09 & 0,21 \\
LOD1.2 & 116,71 & 1,02 & 102,64 & 0,40 & 118,45 & 0,85 \\
LOD1.3 & 117,67 & 0,06 & 103,03 & 0,01 & 119,24 & 0,06 \\
LOD2.1 & 116,75 & 0,98 & 102,65 & 0,39 & 118,50 & 0,80 \\
LOD2.2 & 117,71 & 0,02 & 103,03 & 0,01 & 119,28 & 0,02
\end{tabular}

Measuring the circumference dimensions shows that recalculating the points to smooth the edges with the Catmull Clark modifier in LOD1.2 and LOD2.1 causes the dimensions to deviate from the scan. The accuracy of the measured values decreases by up to $1 \mathrm{~cm}$ for a subdivision level 1 in the bust circumference. It is also noticeable that the dimensional difference of LOD1.3 and LOD2.2 to the target is only $0.06 \mathrm{~cm}$ and $0.02 \mathrm{~cm}$ respectively. Quadrupling the polycount improves the value by only 0.04 $\mathrm{cm}$.

After the dimensional verification, the area sizes of the segmented body regions were determined for the created LODs.

Diagram 1. Distribution of the surface area

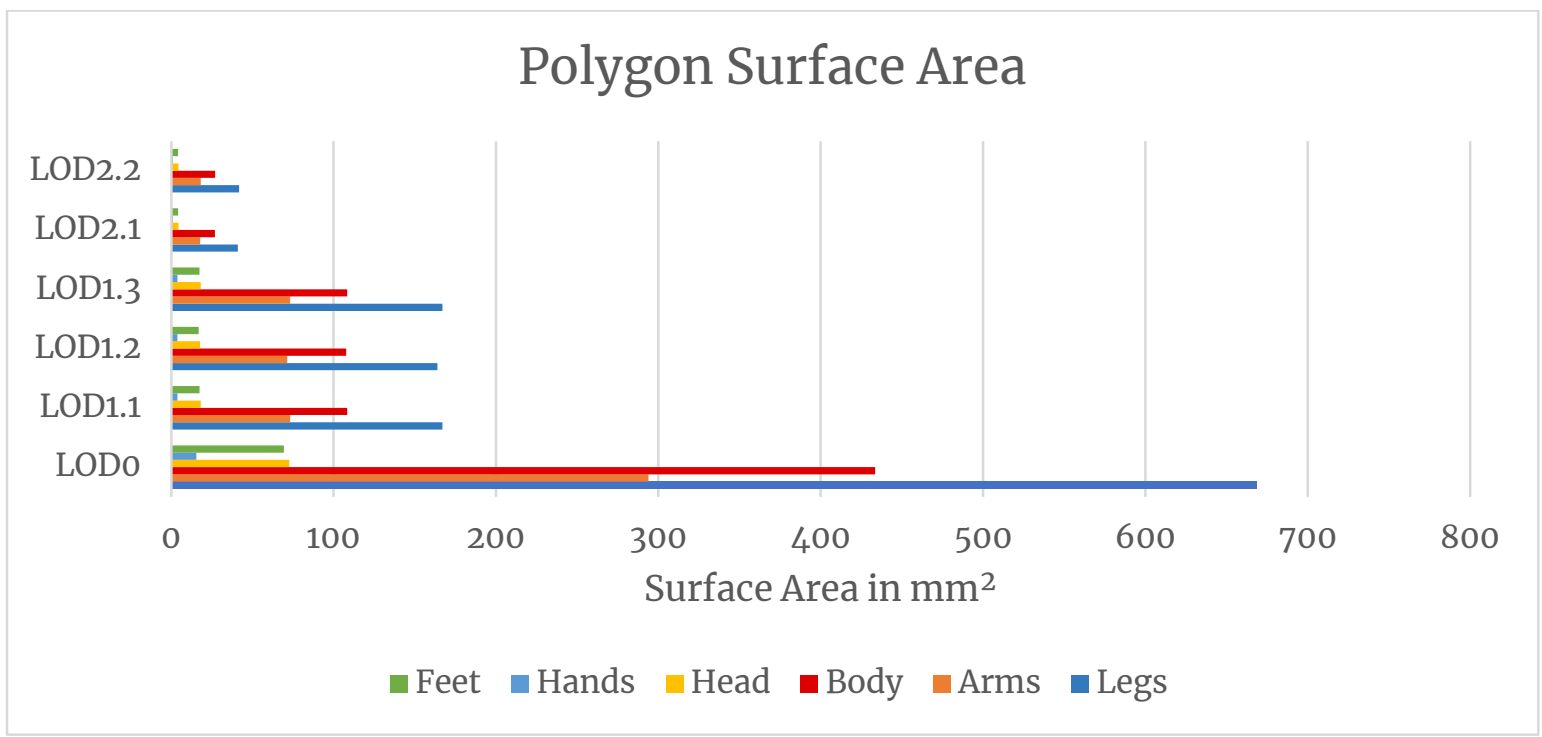

The analysis of the surface dimensions shows how heterogeneously the mesh is constructed. The largest average surface areas of the polygons are on the leg and the smallest on the hands.

\subsection{Virtual Fit}

Since without a re-projection of the points the primary dimensions deviated too much, the fit analysis was only performed on the base avatar LOD0 and on the avatars of the test series whose vertices were projected onto the scan geometry after subdivision, LOD1.3 and LOD2.2.

\section{Visual Fit Inspection}

In this analysis, the visual change in fit was tested via visual aspects. Wrinkles were localized and compared via their direction and position under the simulations. 
The following figures 3 to 5 show the simulated pants German size 50 on the avatars in the systems clo3d, Vidya and VStitcher in front view.

During the visual inspection, the thing that stood out most was that the polygons of the avatar were visible through the trousers at the level of the thighs of avatar LODO in all systems. In Clo3D it is well visible how the wrinkles in the crotch area dissolve the higher the detail level of the avatar becomes.
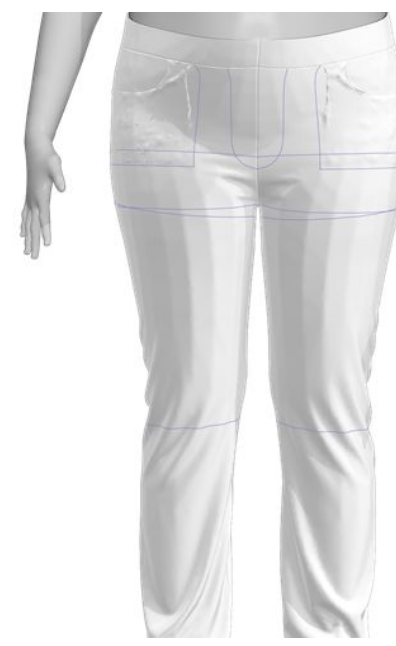
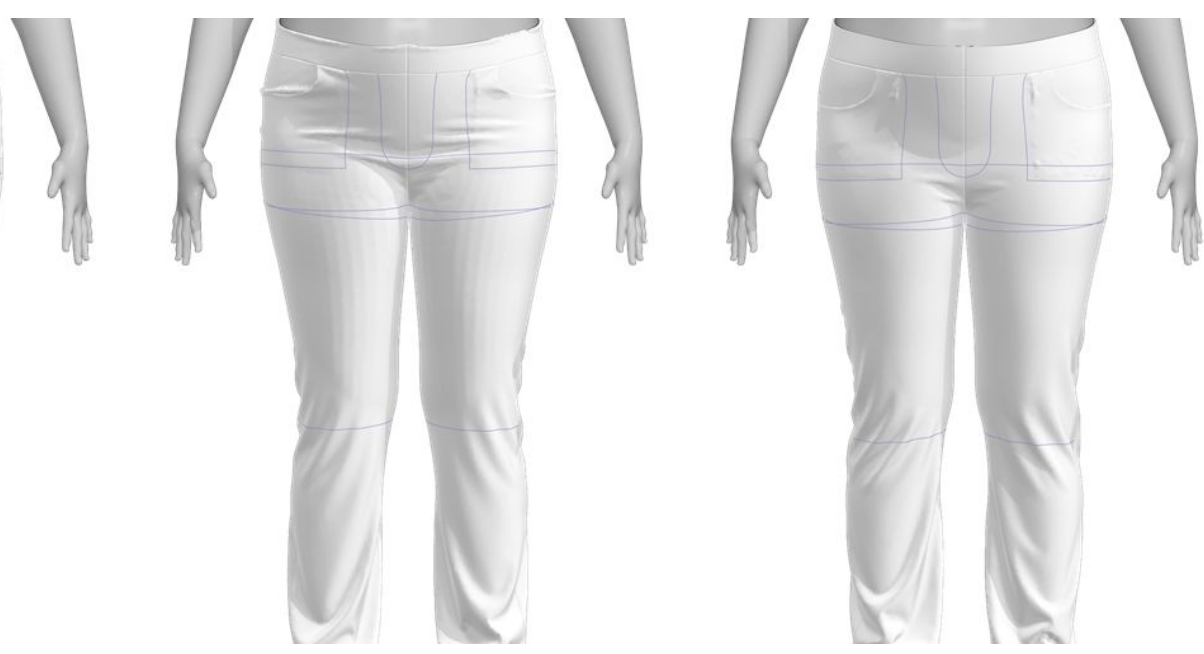

Fig. 3. Trouser simulation Clo3D (f.I.t.r. LODO, LOD1.3, LOD2.2)

The simulation results of the avatar series in Vidya also show that with a higher resolution of the avatars, the polygons no longer show on the clothing. A clear change in fit via the change in wrinkle shape or position cannot be visually determined with certainty. The greatest visual change takes place between avatar LOD0 and LOD1.3.
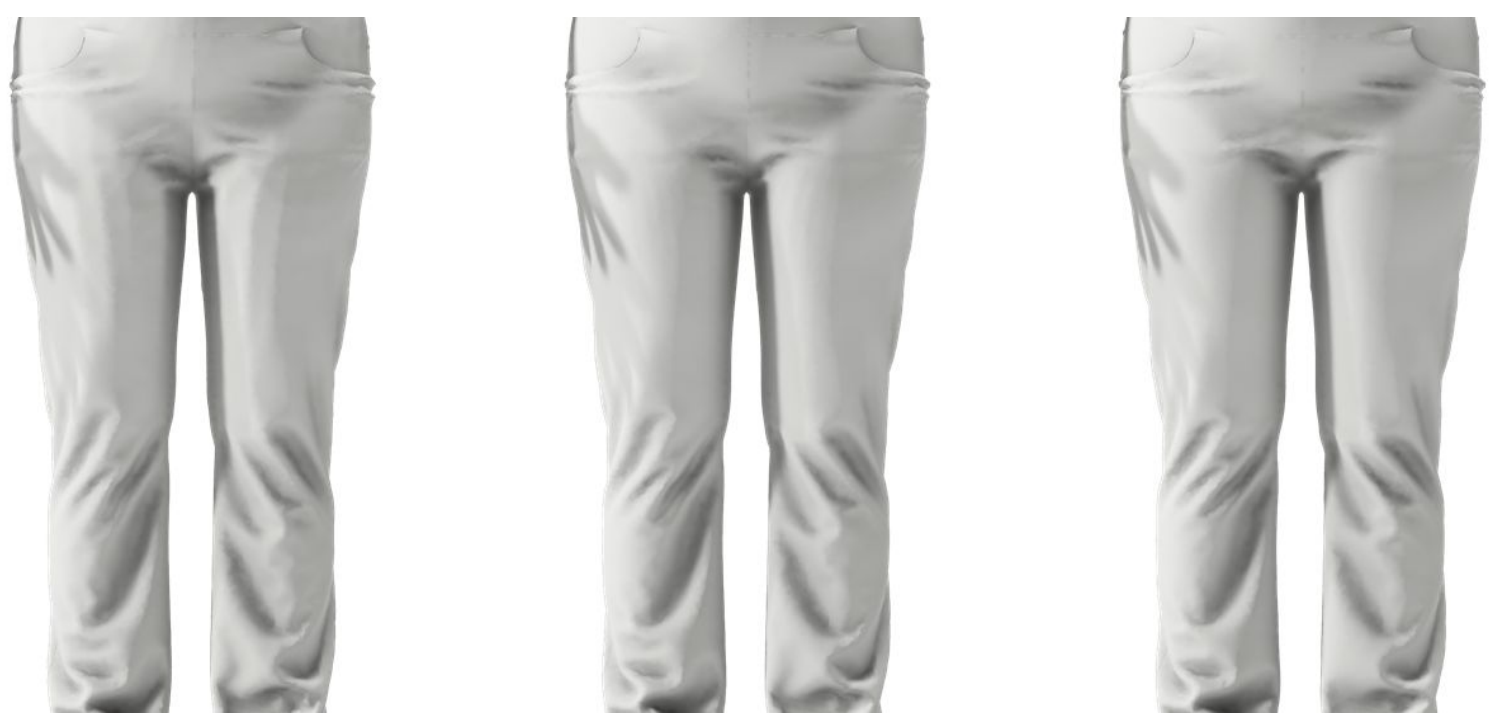

Fig. 4. Trouser simulation Vidya (f.l.t.r. LODO, LOD1.3, LOD2.2) 
Similarly to Vidya, the greatest visual change in the fit of the trousers occurs between the simulations on avatars LOD0 and LOD1.1. The structure of the avatar is already barely visible in LOD1.3.
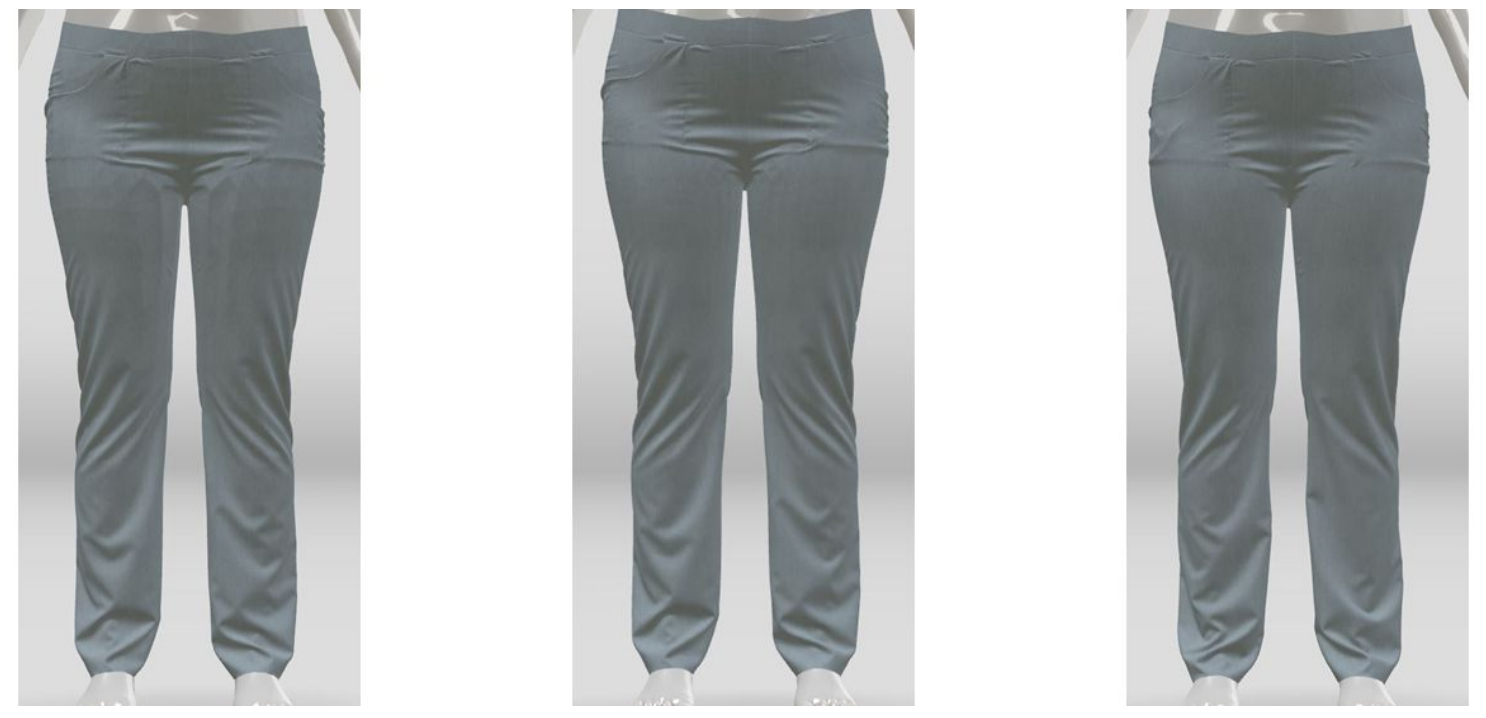

Fig. 5. Trouser simulation VStitcher (f.I.t.r. LODO, LOD1.3, LOD2.2)

In all simulation systems, it was particularly noticeable that the polygons of the avatar were visible on the clothing. With the size of the grid of the cutting geometries in the simulation, it was possible to analyse how large the area of the avatar's polygons were where the artefacts no longer occurred by analysing the grid structure of the avatar beforehand. For this purpose, it was calculated how many points of the pattern geometry were located on an average area of the avatar's leg.

Table 5. Average point density of simulated geometries per area of the avatar on the leg for the Clo3D, Vidya and Vstitcher systems.

\section{Av point density on av. Polygon surface leg}

$\begin{array}{lllll}\text { System } & \text { Grid size in } \mathrm{mm} & \text { LOO } & \text { LOD1 } & \text { LOD2 } \\ \text { Clo3D } & 5 & 26.74 & 6.68 & 1.64 \\ \text { Vidya } & 7.36 & 12.34 & 3.08 & 0.76 \\ \text { Vstitcher } & 2 & 167.10 & 41.77 & 10.26\end{array}$

\section{Structural Fit Inspection}

The following figures 3 to 5 show the simulated pants German size 50 on the avatars in the systems clo3d, Vidya and VStitcher in front view with the tension maps.

In this analysis, the tools of the systems were used to show the tension of the material on the cutting geometries via a colour gradient. In all systems, the colour blue stands for no stress and a colour gradient from green, yellow to the maximum measured elongation indicated by red for an increase in tension. The change in the colour gradients gave an indication of the influence of the resolution of the avatar geometry on the simulation. 
In Clo3D, the strength of the measured tension changed greatly across the avatars. In the simulation on avatar LOD0, a tension at the level of the upper part of the thighs was indicated in red, but this decreased over the simulation series and was only shown as yellow/green on avatar LOD2.2.
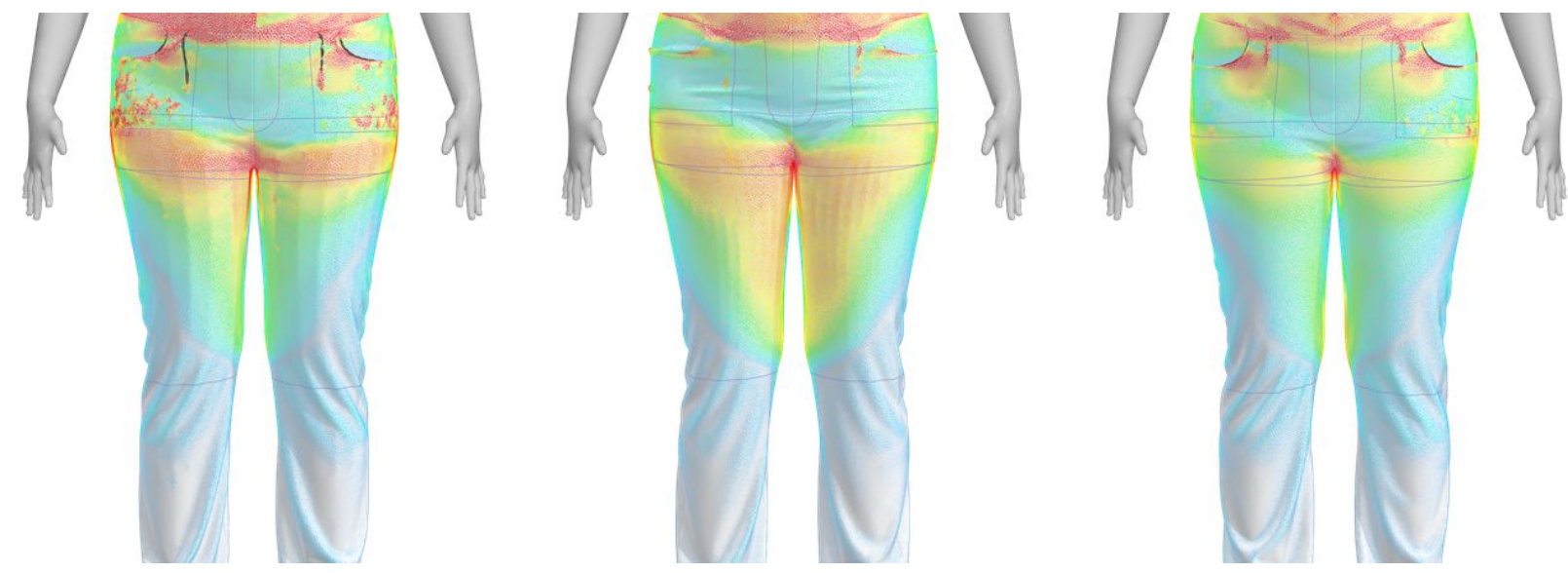

Fig. 6. Display of the tension map calculated in Clo3D (f.I.t.r. LOD0, LOD1.3, LOD2.2)

In Vidya, a change in tension could be noticed in the crotch area. In the simulation on avatar LOD0, a larger range without tension was measured in the crotch area than in the simulation on avatar LOD2.2.
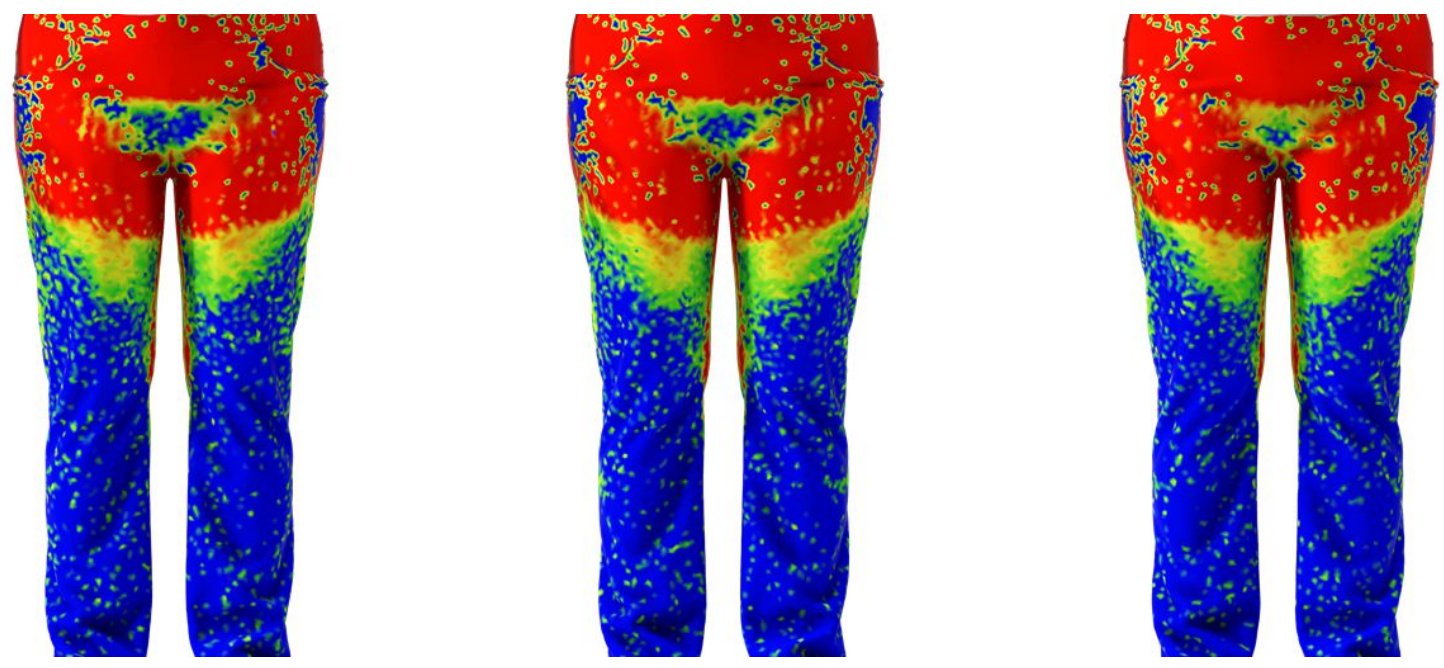

Fig. 8. Display of the tension map calculated in Vidya (f.I.t.r. LODO, LOD1.3, LOD2.2)

In VStitcher, the distribution of the tension fields and also the measured tension was comparable across all simulations. Only in the crotch area was a change recognisable; in the simulation on Avatar LOD0, a tension was still measurable in this area, but no longer in the other two simulations.
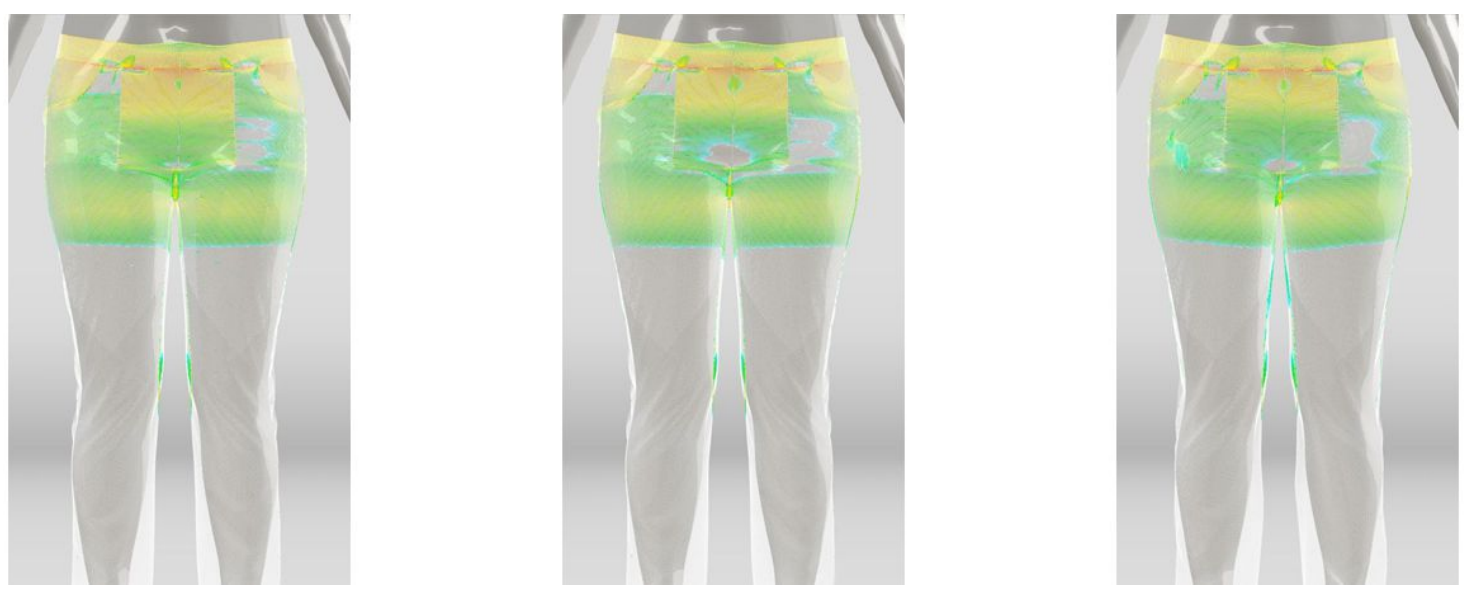

Fig. 7. Display of the tension map calculated in VStitcher (f.I.t.r. LODO, LOD1.3, LOD2.2) 


\section{Conclusion}

Simulation programmes are used more and more in garment development processes. They can already be used to check the fit in a digital environment. Yet, to understand the output a comprehensive knowledge of traditional fit assessment and of the new tools is necessary. Digital fit is influenced by multiple factors which need to be investigated in the future successively. Avatars are the basis for digital processes. Therefore, research in this field is essential. Different Level of Detail are possible to be utilized in each simulation software. Starting from LOD0 the number of polygons is increasing from level to level many times over. Though, the influence on the clothing simulation was unknown. Only software VStitcher provides a recommendation regarding the total number of polygons found in a threedimensional model. The research question posed was what influence the resolution of the avatar geometry has on the clothing simulation. In this project three LODs of the same female avatar German size 50 were created. The geometry was investigated, and it was shown that there was no significant influence on the main measurements bust, waist and hip circumference when reprojected. The digital fit assessment with simulated pants indicates that for the visualisation of clothing, a finer grid structure is necessary, because otherwise the surfaces of the avatar geometry are pushed through the closefitting clothing. The optimal definition of the grid size of the avatar still has to be developed, but a first approach is the comparison in Table 6 . This can be used to calculate the optimal size of the polygon areas for the basis of creating a more homogeneous grid structure of the avatar for simulation according to the specifications in Table 2 for a more accurate fit simulation. The virtual fit test revealed differences between the simulations on the created avatars. Yet, only in one system a clear difference in fit could be determined. Whether the fit was better or worse cannot be answered unequivocally. It cannot be excluded that other factors, e. g. material pre-set or garment positioning, had an impact on the fit assessment results. In the future, this should be further examined. In addition, clothes especially for the upper body should be simulated and fitting test with male avatars be performed. Furthermore, it could not be determined how the increased number of polygons affects the simulation speed. This will be tested in further trials via the integration of an animation in the simulation systems.

\section{References}

[1] J. Power, "Fabric objective measurements for commercial 3D virtual garment simulation," International Journal of Clothing Science and Technology, vol. 25, pp. 423-439, 2013, https://doi.org/10.1108/IJCST-12-2012-0080.

[2] S. Krzywinski and J. Siegmund, "3D Product Development for Loose-Fitting Garments Based on Parametric Human Models," presented at the 17th World Textile Conference AUTEX 2017, Corfu (Kerkyra) (GR), 2017, https://doi.org/10.1088/1757-899X/254/15/152006.

[3] S. Morlock, "Passform \& Schnitt im Wandel - Mit 3D-Technologie in die Zukunft," TextilPlus, vol. 09/10, pp. 13-15, 2020,

[4] A. S. M. Sayem, "Virtual fashion ID: a reality check," presented at the IFFTI Conference, 8 -11 April 2019, Manchester Fashion Institute, Manchester (GB), 2019,

[5] Assyst GmbH. (10.09.2021). 3D-Vidya. Available: https://www.assyst.de/de/produkte/3dvidya/index.html

[6] CLO Virtual Fashion LLC. (10.09.2021). CLO. Available: https://www.clo3d.com/

[7] Browzwear Solutions Pte Ltd. (10.09.2021). VStitcher. Available: https://browzwear.com/products/v-stitcher/

[8] I. Alvanon. (10.09.2021). The Virtual AlvaForm. Available: https://alvanon.com/solutions/3d/

[9] A. GmbH. (10.09.2021). 3D-Form. Available: https://www.avalution.net/de/fashion/avatarebuesten/index.html

[10] I. Daz Productions. (10.09.2021). Daz 3D. Available: http://daz3d.com/

[11] I. Epic Games. (10.09.2021). MetaHuman Creator. Available: https://www.unrealengine.com/enUS/digital-humans

[12] T. M. Team. (10.09.2021). MakeHuman. Available: http://www.makehumancommunity.org/

[13] B. S. P. Ltd. Imported Avatar Requirements. Available: https://support.browzwear.com/VStitcher/Avatars/imp-avatar-requirements.htm 